\title{
High Rate of Radiological Failure Despite Excellent Clinical Results of Minimally Invasive Acromio-Clavicular Joint Reconstruction (MINAR)
}

\author{
Marc Banerjee $^{1,2 *}$, Carolin Spüntrup ${ }^{3}$, Bertil Bouillon', Ann-Kathrin Singer ${ }^{1,4}$, \\ Rolf Lefering $^{5}$, Arasch Wafaisade ${ }^{1}$ and Maurice Balke ${ }^{1,6}$ \\ ${ }^{1}$ Department of Trauma and Orthopedic Surgery, University of Witten/Herdecke, Cologne \\ Merheim Medical Center, Germany \\ ${ }^{2}$ Department of Orthopedic Surgery and Sports Traumatology, Atos MediaPark Clinic, Cologne, \\ Germany \\ ${ }^{3}$ Pelvic School Saarbrücken, Germany \\ ${ }^{4}$ Department of Trauma, Orthopaedic and Plastic Surgery, University of Göttingen, Germany \\ ${ }^{5}$ IFOM, Institute for Research in Operative Medicine, University of Witten/Herdecke, Cologne \\ Merheim Medical Center, Germany \\ ${ }^{6}$ Sportsclinic Cologne, Cologne, Germany \\ *Corresponding Author: Marc Banerjee, Department of Trauma and Orthopedic Surgery, \\ University of Witten/Herdecke, Cologne Merheim Medical Center and Department of \\ Orthopedic Surgery and Sports Traumatology, Atos MediaPark Clinic, Cologne, Germany.
}

Received: September 28, 2020

Published: October 30, 2020

(C) All rights are reserved by Marc

Banerjee., et al.

\section{Abstract}

Introduction: Hook plates and arthroscopic flip button techniques are currently the most commonly used procedures for AC-joint reconstruction. Minimally invasive AC-joint reconstruction (MINAR), a mini open flip button technique was introduced as an alternative to the arthroscopic technique as it is a straight forward procedure and no experience in arthroscopy is needed.

Methods: Between 2011 and 2015, 53 patients with acute AC-joint separations Type Rockwood III, IV and V were stabilized by MINAR. 45 were available for follow-up. In 15 patients a $1 \mathrm{~m}$ m polyester suture was used, in the remaining 30 patients a No. 5 Fiberwire. All patients had a follow-up examination at a minimum one year after surgery. The Constant score (CS), the American Shoulder Elbow score (ASES) and the subjective shoulder value (SSV) were evaluated. All patients had a calibrated bilateral stress view of the AC-joints and a bilateral axial view. The coraco-clavicular (cc) distance was measured on the injured and contralateral side. Furthermore, the width of the clavicular tunnel was measured and compared with the initial diameter (4.5 mm).

Results: The mean age of the patients was 37.6 years (range 17 - 64), patients were operated after an average of 7.2 (range 1 - 20 ) days after trauma. After a mean of 25.3 months (range 12 - 53 months) the mean absolute CS was 96.6 (range 87 - 100 ), the mean ASES 98.0 (range 60 - 100) and the mean SSV was 92.3 (range 60 - 100). The cc-distance was $10.0 \mathrm{~mm}$ on the injured side and $8.1 \mathrm{~mm}$ on the uninjured side $(\mathrm{p}<0.001)$. Only 14 patients $(31.1 \%)$ had a perfect reduction in the vertical plane defined as a cc-distance on the injured side of $\pm 10 \%$ compared to the contralateral side. $20 \%$ of the patients with polyester suture and $56.7 \%$ of patients with Fiberwire had a widening of the clavicular tunnel with an average tunnel of $3.5 \mathrm{~mm}$ and $5.0 \mathrm{~mm}, \mathrm{p}=0.016$.

Conclusion: Despite excellent clinical results and an average side-to-side difference of the cc-distance comparable to other techniques MINAR failed to restore the AC joint anatomically in the vertical plane. Therefor a modification of the originally described technique is advisable.

Keywords: Acromioclavicular Joint Dislocation; Acromioclavicular Joint Reconstruction; Coracoclavicular Stabilization; MINAR 


\section{Introduction}

Several techniques for the reconstruction of acute acromio-clavicular (AC) joint injuries have been described [1,2]. Currently besides hook plates coraco-clavicular stabilization techniques using flip button constructs are most commonly used [3]. A disadvantage of hook plates is the need for a second operation as removal is mandatory. Furthermore, the procedure is not minimally-invasive and a loss of correction or recurrence of dislocation has been described [4-6]. In contrast to suspensory devices like the tight-rope technique or other flip button techniques a higher complication rate was reported in a current review [2].

Coraco-clavicular stabilization procedures have been advocated as they can be performed either arthroscopically or minimally-invasive. The clinical results reported after arthroscopic acromio-clavicular joint stabilization are excellent. Radiologically a side-to-side difference in the coraco-clavicular distance has been shown at the time of follow-up caused by a loss of reduction [7-9]. Thus, it is recommended that arthroscopically assisted stabilization should be performed by a specialist to avoid loss of reduction and to minimize the complication rate. As AC joint injuries are common injuries operation by a specialist is not always possible. Petersen., et al. introduced a minimally-invasive AC-joint reconstruction (MINAR) in which a flip-button construct is placed between the undersurface of the coracoid and the surface of the lateral clavicle using specifically developed instruments for minimally invasive placement [10]. Beside the advantage of a minimalinvasive procedure the technique is straight forward and can also be performed by surgeons not specialized on shoulder surgery. Excellent clinical results have been reported [11] with no difference to the results of hook plate [12]. As with other techniques MINAR showed a loss of reduction. Furthermore, a revision rate of 10.8 $14.3 \%$ was reported $[11,13]$.

As the current literature on the MINAR exclusively comes from the group that developed the technique, the current study intended to report independent results on the clinical and radiological outcome following minimal-invasive AC joint reconstruction. Our hypothesis was that excellent clinical and radiological results can be achieved.

\section{Patients and Methods}

Between 2011 and 2015, 53 consecutive patients with acute Rockwood III to V injuries underwent minimally-invasive AC joint reconstruction (MINAR). Surgery was performed by the first author or in the presence of the first author. Acute injury was defined as an interval between trauma and operation of at most three weeks. All patients had standard radiographs preoperatively (bilateral stress view of the AC-joints loaded with $10 \mathrm{~kg}$ and axial view) on which classification according to Rockwood was made. In patients with Rockwood III injuries conservative and operative treatment was discussed with the patient and indication for surgery was made on an individual basis. In all patients with Rockwood IV and V injuries surgery was advised.

Patient' demographics, operative time and complications as well as reoperations were taken from patient's records.

\section{Operative technique}

Surgery was carried out with the patients in the beach chair position. A 3 to $4 \mathrm{~cm}$ sabre cut incision was made $3 \mathrm{~cm}$ medial to the AC joint. The fascia was incised in a longitudinal direction. The lateral clavicle was exposed and anterior to the clavicle a tunnel was created down to the coracoid process. The fascia lateral to the coracoid was incised and the aiming device was placed underneath the coracoid. A $2.4 \mathrm{~mm}$ K-wire was drilled through the coracoid close to the base and after the correct position was palpated the wire was overdrilled with a $4.5 \mathrm{~mm}$ drill. The first button of the prepared flip button construct consisting of two flipptacks (Karl Storz, Tuttlingen, Germany) and a No. 5 Fiberwire (Arthrex, Naples, Florida, U.S.A.) or a $1 \mathrm{~mm}$ Ethibond suture (Ethicon, Norderstedt, Germany) was brought through the drill hole with a button pusher. The button was flipped underneath the coracoids process by pulling on the thread. A $4.5 \mathrm{~mm}$ drill hole was made into the lateral clavicle with the aiming device in the same manner and the second button was brought through the drill hole. The button was positioned on the upper side of the clavicle. The AC joint was reduced under radiographic control and the zip loop construct was fixed by surgical knots. The trapezoidal and deltoid fascia was closed before subcutaneous and skin closure.

After surgery the shoulder was placed in a sling for 4 weeks. Passive mobilisation with an abduction and flexion up to $45^{\circ}$ was recommended for the first 3 weeks and up to $90^{\circ}$ for the next three weeks. An active free range of motion was allowed after 6 weeks. Lifting, activities stressing the AC joint and return to sports were allowed after 4 months. 


\section{Clinical and radiological follow-up}

At final follow-up all patients had a complete physical examination of both shoulders. The AC joint was evaluated regarding vertical or horizontal instability and AC joint tenderness. Furthermore, the knot and button on the clavicle were palpated regarding tenderness.

In all patients the Simple Shoulder Test (SST), the Subjective Shoulder Value (SSV), the American Shoulder and Elbow Surgeons Score (ASES) and the absolute Constant Score (CS) were evaluated. Satisfaction with the result was evaluated on a 10 point scale $(0$ highest dissatisfaction, 10 maximum satisfaction) while pain was evaluated on a Visual Analogue Scale (VAS) with 0 indicating no pain and 10 indicating highest possible pain.

All patients had a bilateral stress view with $10 \mathrm{~kg}$ on each side and an axial radiograph of both shoulders with a calibrated measuring device for compensation of the magnification factor. On these radiographs the coraco-clavicular distance and the width of the clavicular bone tunnel in the mid of the clavicle were measured. In the axial views the horizontal position of the AC joint was evaluated. To quantify the final radiological outcome in the vertical plane the percentage of the CC distance of the affected side compared to the unaffected side was calculated. The value was evaluated according to the Rockwood classification and the Acromioclavicular Joint Injury score (ACJI) plate [14]. A side to side difference of less than $10 \%$ was regarded as a perfect radiological result in the vertical plane. If the difference was 10 to $25 \%$ a result according to a type II Rockwood injury was achieved. If the CC difference was between 26 and $100 \%$ the result was similar to a type III injury. If a difference of more than $100 \%$ was present the result is similar to a Rockwood V injury. Overcorrection was defined as side to side difference in the cc distance of the affected side to the unaffected side exceeding $10 \%$.

Patients gave their informed consent and permission to participate in the study and institutional approval for the study was granted by the Medical Review Board of our institute (trial number 134/2013).

\section{Statistical analysis}

Statistical analysis was performed using SPSS 20 (SPSS Inc, Chicago, Illinois). A p-value $<0.05$ was considered significant (Fishers exact $t$-test).
Results

After a mean of 25.3 months (range 12 - 53 months) 45 patients were available for follow-up. 8 patients were lost to follow-up, 3 of them had moved away and 5 refused participation in the study because they were free of symptoms and felt that an examination was not necessary. There was one woman and 44 men. The mean age of the patients was 37.6 years (range 17 - 64 years). According to the Rockwood classification there were 13 type III, 8 type IV and 24 type $V$ injuries. The mean time between trauma and surgery was 7.2 days (range 1 - 20 days). The mean operation time was 45 minutes (range 23 - 77 minutes).

\section{Clinical and radiological outcome}

At follow-up the injured side showed a mean CS of 96.6 (range 87 - 100), a mean ASES of 98.0 (range 60 - 100) and a mean SSV of 92.3 (range 60 - 100). The corresponding values for the contralateral side were 98.2 (range 91 - 100) for the CS, 99.8 (range 95 - 100) for the ASES and 99.6 (range 98 - 100) for the SSV. The differences were statistically significant for all three scores $(p=0.002$, $p=0.004$ and $p<0.001)$. The mean satisfaction with the result of surgery was 8.9 (range 7 - 10).

The mean absolute (calibrated) cc distance on the injured side was $10.0 \mathrm{~mm}$ (range 3.5 - $17.2 \mathrm{~mm}$ ) and $8.1 \mathrm{~mm}$ (range 3.3 - 10.9 $\mathrm{mm}$ ) on the contralateral side. The difference was statistically significant $(\mathrm{p}<0.001)$.

Patients operated with Fiberwire thread had a postoperative non-stressed bilateral antero-posterior view. In these patients the loss of correction was calculated as the cc difference on the postoperative radiographs compared with those at follow-up (the uncalibrated value). The mean loss of correction was $5.5 \mathrm{~mm}$ (range $0-9.7 \mathrm{~mm}$ ). Loss of correction could not be calculated for patients with Ethibond suture as these patients had no postoperative bilateral radiographs.

20 patients $(44 \%)$ had a radiological failure in the vertical plane defined as a cc difference of more than $25 \%$ compared to the uninjured side. Two of these patients had a cc difference of more than $100 \%$ according to a Rockwood V injury. 14 patients (31.1\%) showed a perfect reduction in the vertical plane (See figure 1). The ASES and Constant score and the Subjective Shoulder Value did not differ in patients with perfect reduction and those with over- or under correction (See diagram 1). 


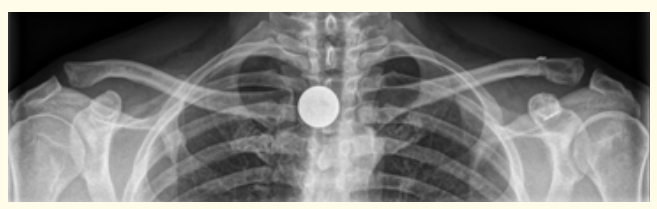

Figure 1: Minimally invasive AC joint reconstruction (MINAR) with perfect reduction in the vertical plane.

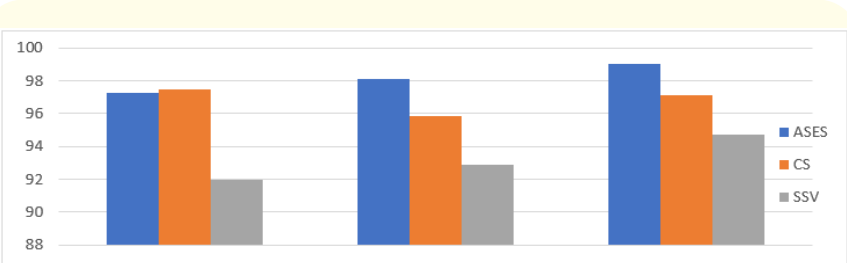

Diagram 1: ASES, CS and SSV in patients with perfect reduction, under- and overcorrection.

12 patients (26.7\%) had a mild (less than one fourth of clavicular width) residual horizontal displacement of the clavicle. In one patient a persistent posterior dislocation was present on the axial follow-up x-ray (See figure 2). A more medial or lateral placement of the clavicular tunnel (the distance of the clavicular tunnel to the ac joint) did not correlate to the calibrated cc distance at follow-up (Pearson correlation coefficient $=0.11$ ).

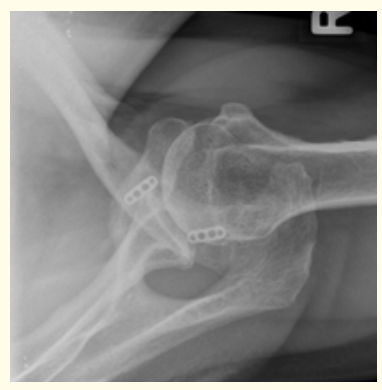

Figure 2: Persistent posterior dislocation of the lateral clavicle.

$27 \%$ of the patients with an Ethibond thread and $57 \%$ of patients with a Fiberwire thread showed a widening of the clavicular tunnel. The average tunnel width was $3.5 \mathrm{~mm}$ for patients with
Ethibond and $5.0 \mathrm{~mm}$ for patients with Fiberwire $(\mathrm{p}=0.016)$. Due to the study design patients with Ethibond threads had a longer follow-up than patients with Fiberwire (39.5 vs 18.2 month, p < 0.05). Patients with Fiberwire thread that had a tunnel diameter of more than $4.5 \mathrm{~mm}$ at the time of follow-up and patients with a tunnel diameter of $4.5 \mathrm{~mm}$ or less had no statistically significant differences in loss of correction (5.6 vs. $5.3 \mathrm{~mm}, \mathrm{p}=0.199$ ).

At the time of follow-up 10 patients showed an absolute tunnel widening of more than $1 \mathrm{~mm}$ compared to the initially drilled hole (average $6.6 \mathrm{~mm}$, range $5.6-8.9 \mathrm{~mm}$, see figure 3). In 9 out of 10 patients a Fiberwire had been used. The mean cc-distance for patients with Ethibond and Fiberwire thread was not significantly different (10.5 vs. $9.8 \mathrm{~mm}, \mathrm{p}=0.067$ ).

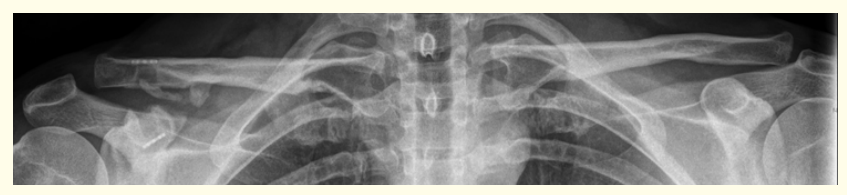

Figure 3: Persistent posterior dislocation of the lateral clavicle.

\section{Complications and re-operations}

One patient sustained a fracture of the coracoid process six weeks after surgery with dislocation of the coracoidal button and complete loss of reduction (See figure 4). The patient was treated conservatively. At the time of follow-up one year after surgery the calibrated cc distance was $16.1 \mathrm{~mm}$ and $8.9 \mathrm{~mm}$ on the uninjured side. The clinical result was moderate (CS 88, ASES 95, SSV 60) despite a high satisfaction of 9 . In four patients the clavicular button had migrated into the clavicula (See figure 5). In two patients the clavicular button and the thread had to be removed because the patients complained local irritation. Another patient had a plastic correction of the scar.

\section{Discussion}

In the current study minimally-invasive AC-joint reconstruction (MINAR) showed excellent clinical, but moderate radiological results. $44 \%$ of the patients had a radiological failure in the vertical plane defined as a cc difference of more than $25 \%$ compared to 


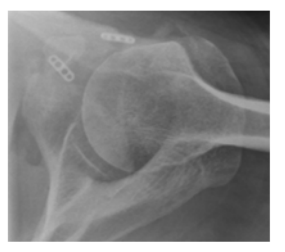

a

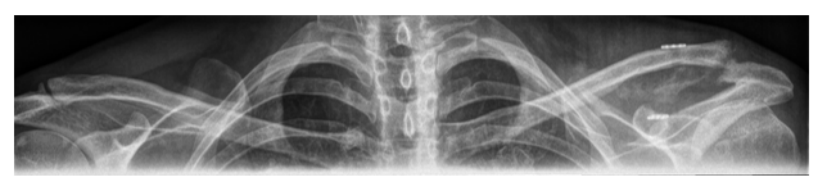

b

Figure 4: Coracoid fracture (a) with dislocation of the coracoidal button and complete loss of reduction (b).

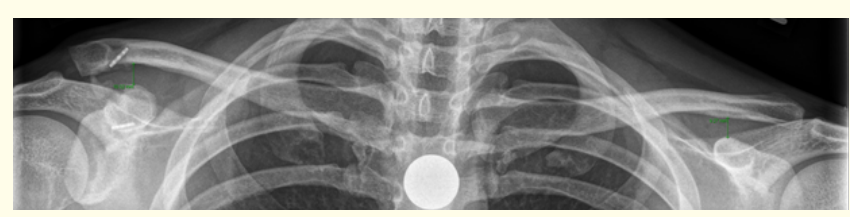

Figure 5: Migration of the clavicle button in the clavicle.

the uninjured side although this had no influence on the clinical outcome. Only one third of the patients had an anatomical ac-joint reduction in the vertical plane. The average cc-distance on the injured side was $10.0 \mathrm{~mm}$ vs. $8.1 \mathrm{~mm}$ on the contralateral side. The mean loss of reduction was $5.5 \mathrm{~mm}$ for patients with No. 5 Fiberwire.

The only clinical outcome study on MINAR comes from the group that introduced the technique.

Rosslenbroich., et al. reported on 83 patients with minimally invasive AC-joint reconstruction [11]. Clinical results were excellent with a mean CS of 94.7. However, nine patients (10.8\%) underwent revision surgery, eight of them due to recurrent dislocation. At the time of follow-up $21.6 \%$ showed radiological failure, defined as loss of reduction of more than $50 \%$ of the clavicular shaft width in the vertical plane. Radiological failure did not influence the clinical result. The cc-distance was not reported.

Gowd., et al. showed in their review of operative techniques for ACJ reconstructions that EndoButton techniques had an average failure rate of $18.3 \%$ [1]. In comparison the most commonly used arthroscopic technique (the tight rope technique) had a failure rate of $20.5 \%$. The main problem in the interpretation of failure rates in different studies is, that there is no common definition for failure as many studies either did not report criteria for radiological failure or only reported loss of reduction without further specification. Studies that specified failure used several definitions (CC distance $>50 \%$ of clavicular width or $>25 \%$ compared with postoperatively and many others) [1]. As in the studies by Vascellari., et al. [15] and Takase and Yamamoto [16] in the current study failure was defined as a difference in the CC-distance of more than $25 \%$ compared to the contralateral side according to a Rockwood III injury in the acute setting as this type is the lowest grade in which surgery is considered.

The reasons for failure of MINAR in the current study are hypothetical. As the clavicular and the coracoidal bone tunnel are drilled separately in the MINAR technique they are not always in line. Thus, the sutures may rub against the bone rim of the tunnels and can tear. In only one patient a fracture of the coracoid was responsible for the failure.

Schliemann., et al. analysed risk factors for failure of the MINAR technique [13]. 9 out of 63 patients (14.3\%) had revision surgery, in eight cases because of recurrent instability. In six patients there was a malpositioning of the coracoid tunnel too far laterally and in two patients too far anteriorly with dislocation of the coracoidal button. Only one of these patients had a complete coracoid fracture. At final follow-up the mean cc-distance was $11.1 \mathrm{~mm}$ on the affected and $9.7 \mathrm{~mm}$ on the contralateral side. Five patients had a complete loss of reduction according to a Rockwood V injury.

Another possibility for failure of MINAR is that a single implant with two buttons and a non-absorbable suture is too weak for ACjoint reconstruction. Breuer, et al. used a double implant MINAR technique with one coracoid button and two clavicular buttons with two sutures. There was an average loss of reduction of $2.1 \mathrm{~mm}$ in the cc-distance at follow-up. $59 \%$ of the 65 patients did not have any loss of reduction [17].

Several studies reported on results of the arthroscopic double tight rope techniques. In the study of Scheibel., et al. there was a loss of reduction in the CC-distance of $4.7 \mathrm{~mm}$ between surgery and follow-up. At follow-up the mean cc-distance on the affected side was $13.6 \mathrm{~mm}$ versus $9.4 \mathrm{~mm}$ on the contralateral side [9]. Salzmann., et al. reported on 23 patients with arthroscopic double tight rope reconstruction. 6 month after surgery the cc-distance was 9.3 $\mathrm{mm}$ and increased to $10.5 \mathrm{~mm} 24$ month after surgery. At that time the cc-distance of the unaffected side was $10.6 \mathrm{~mm}$ [8]. Glanzmann., et al. reported on 19 patients with Rockwood III and IV injuries with a minimum follow-up of 24 month after surgery. At the time of follow-up the difference of cc-distance between both shoulder was $2 \mathrm{~mm}$. There was a loss of reduction of $3.0 \mathrm{~mm}$. Failure, defined as a cc-distance of more than $2 \mathrm{~mm}$ occurred in 6 patients (32\%) [7]. 
Due to the fact that we performed only axial radiographs at follow-up there is no information regarding dynamic posterior translation of the clavicle. We had one complete static posterior dislocation, in 12 others there was a partial horizontal dislocation of the clavicle. For arthroscopically assisted AC-joint reconstruction it has been shown that the addition of a horizontal cerclage could reduce the number of patients with persistent dynamic posterior translation [18] despite there is no consensus regarding the management of horizontal instability [19].

\section{Limitations of the Study}

The design of the present study is retrospective. Second the mean follow-up of 2 years is relatively short. Of the initially operated 53 patients eight patients were not included in the followup which means a dropout rate of $15 \%$. As only axial radiographs were made at the time of follow-up dynamic horizontal instability could not be detected.

\section{Conclusion}

Despite minimally invasive AC joint reconstruction is a straight forward technique that obtained excellent clinical results, the radiological results were unsatisfactory. Thus, a modification of the technique, for example with a second implant must be considered.

\section{Bibliography}

1. Gowd AK., et al. "Current concepts in the management of acromioclavicular dislocations. A systematic review and metaanalysis of operative techniques". American Journal of Sports Medicine 47 (2019): 2745-2758.

2. Moatshe G., et al. "Acromioclavicular and coracoclavicular Ligament reconstruction for acromioclavicular joint instability: a systematic review of clinical and radiographic outcomes". Arthroscopy 34 (2018): 1979-1995.

3. Balke M., et al. "Acute acromioclavicular joint injuries: Changes in diagnoses and therapy over the last 10 years". Unfallchirurg 18 (2015): 851-857.

4. Eschler A., et al. "Hook plate fixation for acromioclavicular joint separations restores coracoclavicular distance more accurately than PDS augmentations, however represents with a high rate of acromial osteolysis". Archives of Orthopaedic and Trauma Surgery 132 (2012): 33-39.

5. Francesco AD., et al. "The use of hook plate in type III and V acromio-clavicular Rockwood dislocation: Clinical and radiological midterm results and MRI evaluation in 42 patients". Injury 43 (2012): 147-152.
6. Heideken J., et al. "Acute surgical treatment of acromioclavicular dislocation type $\mathrm{V}$ with a hook plate: superiority to late reconstruction". Journal of Shoulder and Elbow Surgery 22 (2013): 9-17.

7. Glanzmann MC., et al. "Clinical and radiological results after double flip button stabilization of acute grade III and IV acromioclavicular joint separations". Archives of Orthopaedic and Trauma Surgery 133 (2013): 1699-1707.

8. Salzmann GM., et al. "Arthroscopically assisted 2-bundle anatomical reduction of acute acromioclavicular joint separations". American Journal of Sports Medicine 38 (2010): 11791187.

9. Scheibel M., et al. "Arthroscopically assisted stabilisation of high-grade acromioclavicular joint separations". American Journal of Sports Medicine 39 (2011): 1507-1516.

10. Petersen W., et al. "Minimally invasive acromioclavicular joint reconstruction (MINAR)". Operative Orthopädie und Traumatologie 22 (2010): 52-61.

11. Rosslenbroich SB., et al. "Minimally invasive acromioclavicular ligament reconstruction with a flip button technique (MINAR)". American Journal of Sports Medicine 43 (2015): 1751-1757.

12. Metzlaff S., et al. "Surgical treatment of acute acromioclavicular joint dislocations: hook plate versus minimally invasive reconstruction". Knee Surgery, Sports Traumatology, Arthroscopy 24 (2016): 1972-1978.

13. Schliemann B., et al. "Why does minimally invasive coracoacromial ligament reconstruction using a flip button repair technique fail? An analysis of risk factors and complications". Knee Surgery, Sports Traumatology, Arthroscopy 23 (2015): 14191425 .

14. Rockwood C. "Injuries in the acromioclavicular joint: subluxations and dislocations around the shoulder". In: Rockwood C jr, Green D, eds. Fractures in adults. Philadelphia: JB Lippincott (1984): 860-910.

15. Vascellari A., et al. "Clinical and radiological results after coracoclavicular ligament reconstruction for type III acromioclavicular joint dislocation using three different techniques: a retrospective study". Joints 3 (2015): 54-61.

16. Takase $\mathrm{K}$ and Yamamoto K. "Arthroscopic procedures and therapeutic results of anatomical reconstruction of the coracoclavicular ligaments for acromioclavicular joint dislocation". Orthopaedics and Traumatology: Surgery and Research 102 (2016): 583-587. 
17. Breuer R., et al. "Minimally Invasive AC Joint Reconstruction System (MINAR) in modified triple-button technique for the treatment of acute AC Joint dislocation". Journal of Clinical Medicine 8.10 (2019).

18. Hann C., et al. "Combined arthroscopically assisted coracoand acromioclavicular stabilization of acute high-grade acromioclavicular joint separations". Knee Surgery, Sports Traumatology, Arthroscopy 26 (2018): 212-220.

19. Aliberti G., et al. "Horizontal instability of the acromioclavicular joint. A systematic review". American Journal of Sports Medicine 48 (2020): 504-510.

\section{Assets from publication with us}

- Prompt Acknowledgement after receiving the article

- Thorough Double blinded peer review

- Rapid Publication

- Issue of Publication Certificate

- High visibility of your Published work

Website: https://www.actascientific.com/

Submit Article: https://www.actascientific.com/submission.php

Email us: editor@actascientific.com

Contact us: +919182824667 